\title{
Conservation of angular momentum in thermomagnetic torque experiments*
}

\author{
H. Vestner ${ }^{\dagger}$ and T. W. Adair III \\ Department of Physics, Texas A \& M University, College Station, Texas 77843 \\ (Received 3 June 1974)
}

\begin{abstract}
The conservation of orbital angular momentum has the following consequence for Scott-effect experiments: The torque exerted on the outer cylinder is equal in size but opposite in direction to the torque on the inner cylinder. This is true for each of the two contributions to the torque, namely, the bulk and slip contribution.
\end{abstract}

\section{INTRODUCTION}

Recently Wood et al. ${ }^{1}$ reported an experimental study of the conservation of angular momentum in thermomagnetic torque experiments (Scott ef:fect $\left.t^{2}{ }^{3}\right)$. Actually, they found no net torque on an inverted double-walled tube filled with nitrogen and placed in an axial magnetic field; a temperature difference between the inner and the outer wall was maintained. This observation, as well as previous experiments by Scott et al. ${ }^{4}$ indicate that the torque $\vec{\tau}_{a}$ exerted on the outer cylinder (in a Scott-effect apparatus) is equal in size but opposite in direction to the torque $\vec{\tau}_{i}$ on the inner cylinder, a result which is not surprising at all, since it is based on the conservation of angular momentum, as is shown in Sec. II of this note. In Sec. III of this note the relation between the flow field and the torque is discussed.

\section{CONSERVATION OF ANGULAR MOMENTUM}

Within the range of validity of the generalized Boltzmann equation for a dilute gas of polyatomic molecules, due to Waldmann and Snider ${ }^{5}$ the pressure tensor $p$ is symmetric, ${ }^{B}$

$$
p_{\nu \mu}=p_{\mu \nu} \text {. }
$$

Then the conservation equation for linear momentum

$$
n \frac{D}{D t} m \overrightarrow{\mathrm{v}}+\vec{\nabla} \cdot p=\overrightarrow{0}
$$

leads to a conservation equation for orbital angular momentum

$$
n \frac{D}{D t}(\overrightarrow{\mathrm{X}} \times m \overrightarrow{\mathrm{v}})+\vec{\nabla} \cdot \overrightarrow{\mathrm{L}}=\overrightarrow{0},
$$

where

$$
L_{\mu \nu}=p_{\mu \mu} \cdot \epsilon_{\nu \nu^{\prime}{ }^{\prime}} x_{\nu^{\prime}}
$$

is the flux of orbital angular momentum, and

$$
\frac{D}{D t}=\frac{\partial}{\partial t}+\overrightarrow{\mathrm{v}} \cdot \vec{\nabla}
$$

is the total (or substantial) time derivative. Hence not only is the total angular momentum conserved but also the orbital and intrinsic angular momentum are conserved separately. The integration of Eq. (2) over any gas volume $V$ bounded by the surfaces $\sigma_{K}$ gives

$$
\frac{d}{d t} \iiint_{V} d V n(\overrightarrow{\mathrm{X}} \times m \overrightarrow{\mathrm{v}})+\sum_{K} \iint_{\sigma_{K}} d \sigma \overrightarrow{\mathrm{n}}_{K} \cdot \overrightarrow{\mathrm{L}}=\overrightarrow{0},
$$

where $\overrightarrow{\mathrm{n}}_{K}$ is the local outer unit normal of the gas at $\sigma_{K}$. The force the gas exerts on the unit area of $\sigma_{K}$ is $\overrightarrow{\mathrm{f}}_{K}=\overrightarrow{\mathrm{n}}_{K} \cdot \overrightarrow{\mathrm{p}}$, and $\overrightarrow{\mathrm{n}}_{K} \cdot \overrightarrow{\mathrm{L}}=\overrightarrow{\mathrm{X}} \times \overrightarrow{\mathrm{f}}_{K}$ is the torque per unit area of $\sigma_{K}$, i.e.,

$$
\vec{\tau}_{K}=\iint_{\sigma} d \sigma \overrightarrow{\mathrm{X}} \times \overrightarrow{\mathrm{f}}_{K}
$$

is the total torque exerted on $\sigma_{K}$. In a steady state, Eq. (3) reduces to

$$
\sum_{K} \vec{\tau}_{K}=\overrightarrow{0}
$$

i.e., there is no net torque on any closed container (volume $V$ ) filled with gas. For the application to a Scott-effect experiment we take as volume $V$ the space between the two coaxial cylinders of length $L$ and radii $R_{i}$ and $R_{a}\left(R_{i}<R_{a}\right)$. Since the bottom and top of this volume give no contribution to the torque, we are left with

$$
\vec{\tau}_{a}+\vec{\tau}_{i}=\overrightarrow{0}
$$

i.e., the torque $\vec{\tau}_{a}$ on the outer cylinder is equal in size and opposite in direction to the torque $\vec{\tau}_{i}$ on the inner cylinder. This fact is a direct consequence of the symmetry of the pressure tensor, or equivalently the conservation of orbital angular momentum.

\section{SLIP FLOW IN THERMOMAGNETIC TORQUE EXPERIMENTS}

The possibility of the existence of an azimuthal flow in the thermomagnetic torque experiments has been discussed since Scott and co-workers dis- 
covered this effect. ${ }^{2}$ The existence of this azimuthal flow is apparent from measurements of Scott et $a l .{ }^{4}$ and Weinstein and Keeney. ${ }^{7}$ Two different mechanisms have been considered. Waldmann $^{8}$ introduced a "thermomagnetic slip" due to an asymmetry in the distribution of polarized molecules reflected from the surface. On the other hand, Levi and Beenakker ${ }^{9}$ pointed out that the transverse component of the heat flux (in a magnetic field) causes a thermal creep along the cylinders. Park and Dahler ${ }^{10}$ presented a kinetictheory-based approach to these slip problems. The ensuing flow field gives rise to a torque which may be called the "slip torque."

Even without any flow of the gas there is a torque due to thermal stresses arising from second-order derivatives of temperatures $(\vec{\nabla} \vec{\nabla} T)$. This "bulk torque" has been calculated by Levi et al. ${ }^{11}$ and a quantitative evaluation is possible with the knowledge obtained from the Senftleben Beenakker effects ${ }^{3}$ of viscosity and thermal conductivity. The bulk torque has the same direction as the observed torque but is greater in magnitude,$^{10-12}$ indicating that the slip flow gives rise to a torque in the opposite direction. This conclusion is confirmed by the direct observation of the thermomagnetic slip effects in experiments on the thermomagnetic pressure difference by Hulsman et al., ${ }^{13}$ which give a method to estimate the slip torque ${ }^{8}$ since the thermal stresses do not exist in a gas between flat plates $(\vec{\nabla} \vec{\nabla} T=0)$.

If the magnetic field direction $\vec{h}$ is parallel to the cylinder axis the flow velocity has only an azimuthal component, which depends on the distance $r$ from the axis. The Navier-Stokes equation (neglecting all inhomogeneous terms arising from the polarizations ${ }^{14}$ ) $\vec{\nabla} \cdot \vec{\nabla} \vec{v}=\overrightarrow{0}$ determines the spatial variation:

$$
\overrightarrow{\mathrm{v}}=\overrightarrow{\mathrm{h}} \times \overrightarrow{\mathrm{u}}(C / r+D r),
$$

where $\overrightarrow{\mathrm{u}}$ is the unit vector in the radial direction. From the viscous pressure tensor $\left(\overrightarrow{\mathrm{p}}_{v}\right)_{S}=-2 \eta(\vec{\nabla} \overrightarrow{\mathrm{v}})_{S}$ [where ()$_{s}$ denotes the symmetric traceless part], the resulting slip torque is calculated:

$$
\vec{\tau}_{i}^{s 1}=-\vec{\tau}_{a}^{s 1}=-4 \pi \eta L C \vec{h} .
$$

This result shows that the slip torque on the outer cylinder is equal in magnitude but opposite to the slip torque on the inner cylinder. Notice that only the $r^{-1}$ term in $\vec{v}$ contributes to $\vec{\tau}_{i}^{s 1}$ and that the gas and the inner cylinder rotate in different directions.

The constants $C$ and $D$ are determined from the boundary conditions at both surfaces. In the highpressure limit $C$ is of the order $1 / p$ and $D$ vanishes ${ }^{10}$ (i.e., is of the order $1 / p^{2}$ ). Consequently, the velocity varies with $r^{-1}$ and is unidirectional.
The bulk contribution $\vec{\tau}_{i}^{b}$ to the total torque

$$
\vec{\tau}_{i}=\vec{\tau}_{i}^{s 1}+\vec{\tau}_{i}^{b},
$$

was calculated by Levi et al. ${ }^{11}$ from the thermal stresses which are determined by $\vec{\nabla} \vec{\nabla} T$.

In Fig. 1 the direction of the flow field and of the resulting slip torque are shown for nitrogen gas in a Scott torque apparatus with a heated cylinder. According to thermomagnetic pressure difference measurements by Hulsman et al., ${ }^{13}$ the gas turns in the $\vec{h} \times \vec{\nabla} T$ direction; hence the slip torque $\vec{\tau}_{i}^{s 1}$ has the direction $\vec{h}$ of the magnetic field. The total torque ${ }^{2}$ as well as the bulk torque ${ }^{12}$ have the opposite direction $-\vec{h}$.

In experiments by Weinstein and Keeney ${ }^{7}$ and by Scott et $a l .{ }^{4}$ radial baffles were introduced between the two cylinders to disturb the flow field. A decrease of the torque was observed in cases where the distance between the baffle edge and the inner cylinder was smaller than about 10 mean free paths. ${ }^{4}$ Such a small clearance interrupts the free circulation of the gas and causes it to reverse its direction at the baffle. As a result of the interplay between this counterflow in the central part of the circular gap and the slip flow along the cylinder walls, a flow field is established such that there is no net gas flow. The velocity gradient near the inner cylinder is thus increased by the presence of a barrier and hence the slip torque (caused by the flow) is also increased. Consequently, the introduction of a baffle causes a reduction in the total torque. This explanation of the "baffle or barrier" experiments, together with a quantitative estimate, was given by Waldmann ${ }^{15}$ as early as 1970.

\section{CONCLUSION}

The symmetry of the pressure tensor, valid for a dilute gas, results in the conservation of orbital

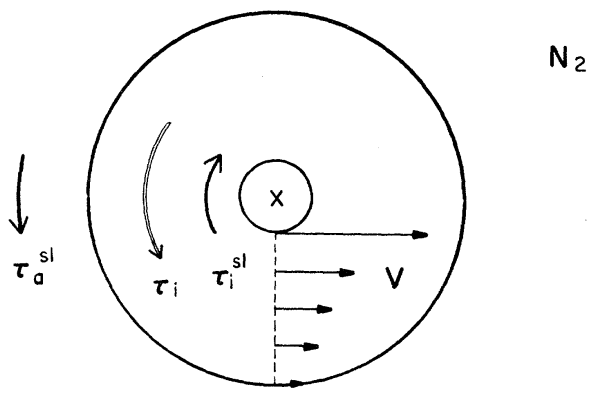

FIG. 1. Directions of the flow field and the torques for $\mathrm{N}_{2}$ in a Scott-torque apparatus with a heated inner cylinder; the magnetic field points into the drawing plane. 
angular momentum. It has been shown that, as a consequence, the torque on the outer cylinder is opposite in direction but equal in magnitude to the torque exerted on the inner cylinder. Since this is true for both the bulk and the slip contributions separately, the assumption of any additional source of angular momentum is not needed in order to explain any of the torque experiments.
*Research supported by The Robert A. Welch Foundation, Houston, Tex.

†Permanent address: Institut für Theoretische Physik der Universität Erlangen-Nürnberg, Erlangen, Germany.

${ }^{1}$ L. T. Wood, C. W. Wells, F. A. Handler, and A. F. Hildebrandt, Phys. Rev. A 9, 2245 (1974).

${ }^{2}$ G. G. Scott, H. W. Sturner, and R. M. Williamson, Phys. Rev. 158, 117 (1967); Phys. Lett. A 25, 573 (1967). T. W. Adair III, D. A. Avery, C. F. Squire, and S. Wolfson, Phys. Rev. Lett. 20, 142 (1968).

${ }^{3}$ For a review of the Scott effect and related phenomena see J. J. M. Beenakker and F. R. McCourt, Annu. Rev. Phys. Chem. 21, 47 (1970).

${ }^{4}$ G. G. Scott, H. W. Sturner, and M. E. Larchez, Phys. Rev. A 2, 792 (1970).

${ }^{5}$ L. Waldmann, Z. Naturforsch. A 12,660 (1957); A 13 609 (1958). R. F. Snider, J. Chem. Phys. 32, 1051 (1960).

${ }^{6}$ S. Hess and L. Waldmann, Z. Naturforsch. A 21, 1529 (1966).
${ }^{7}$ D. H. Weinstein and J. Keeney, Phys. Rev. Lett. 23, 218 (1969).

${ }^{8}$ L. Waldmann, Z. Naturforsch. A 22, 1678 (1967).

${ }^{9}$ A. C. Levi and J. J. M. Beenakker, Phys. Lett. A 25 , 350 (1967).

${ }^{10}$ Won-Hoon Park and J. S. Dahler, J. Chem. Phys. 56, 1995 (1972). J. S. Dahler and Won-Hoon Park, J. Chem. Phys. 56, 4190 (1972).

${ }^{11}$ A. C. Levi, F. R. McCourt, and J. J. M. Beenakker, Physica (Utr.) 42, 363 (1969).

${ }^{12}$ A. L. J. Burgmans and T. W. Adair III, J. Chem. Phys. 59, 324 (1973).

${ }^{13}$ H. Hulsman, F. G. van Kuik, H. F. P. Knaap, and J. J. M. Beenakker, Physica (Utr.) 57, 522 (1972). H. Hulsman, G. F. Bulsing, G. E. J. Eggermont, L. J. F. Hermans, and J. J. M. Beenakker, Physica (Utr.) 72, 287 (1974).

${ }^{14}$ H. Vestner, Z. Naturforsch. A $\underline{28}, 869$ (1973); 28, 1554 (1973).

${ }^{15}$ L. Waldmann, Bull. Am. Phys. Soc. 15, 262 (1970). 